\title{
SEMANTIC CHANGE DETECTION OF RIVER GROUND POINTS IN AIRBORNE LIDAR BATHYMETRY DATA USING VOXEL OCCUPANCIES
}

\author{
R. Boerner*, L. Hoegner, U. Stilla \\ Photogrammetry and Remote Sensing, Technical University of Munich(TUM), Germany \\ - (richard.boerner, ludwig.hoegner, stilla)@tum.de
}

KEY WORDS: bathymetry, change detection, Point clouds, LiDAR

\begin{abstract}
:
This paper proposes a method to get semantic information of changes in bathymetric point clouds. This method aims for assigning labels to river ground points which determine if either the point can be compared with a reference DEM, if there are no data in the reference or if there are no water points inside the new Data of wet areas of the reference data. This labels can be further used to specify areas where differences of DEMS can be calculated, the comparable areas. The Areas where no reference data is found specify areas where the reference DEM will have a higher variance due to interpolation which should be considered in the comparison. The areas where no water in the new data was found specify areas there no refraction correction in the new data can be done and which should be considered with a higher variance of the ground points or there the water surface should be tried to reconstruct. The proposed approach uses semantic reference data to specify water areas in the new scan. An occupancy analysis is used to specify if voxels of the new data exist in the reference or not. In case of occupancy, the labels of the reference are assigned to the new data and in case of no occupancy, the label of changed data is assigned. A histogram based method is used to separate ground and water points in wet areas and a second occupancy analysis is used to specify the semantic changes in wet areas. The proposed method is evaluated on a proposed data set of the Mangfall area where the ground truth is manually labelled.
\end{abstract}

\section{INTRODUCTION}

The common method to look for changes in the area of the river ground is to calculate differences of DEMs and check if the difference is significant. The test of significance is important to distinguish between geometric changes and measurement or registration errors. To create maps of geometric changes of large scale river areas, airborne lidar bathymetrie (ALB) is widely used. The most important classes by working with ALB data are the ground and the water which are needed to know to correct light refraction effects between the medium air and the medium water. If not considered, this refraction effects will result in incorrect measured river ground points. However, the recognition of the water surface or the river ground inside the laser pulse depends on many external conditions, e.g. water turbidity or material of the ground. Therefore, the water segment and river ground segment can differ between multi temporal data. There can be unrecognised water surfaces or ground points which were not measured in the reference data. This paper proposed a method to compare multi temporal ALB data as a pre-processing step for DEM calculation to check for geometric changes. The pre-processing will search for areas where are differences in recognising water surface and river ground points. Considering semantic reference data a new data set can be used to generate labels for unrecognised water pulses inside previous water area, labels for unrecognised ground points in the reference data and labels for areas which can be compared using the difference of DEMs. This labels are important for the search of significant changes because the points in the labelled areas should be handled in different ways. If there is no recognition of the water surface, there can no refraction correction be calculated and it should either be tried to reconstruct the water surface based on surrounding measurements or the variance of the measured ground point should be considered as higher variances. If there is no measurement of the river ground in the refer- ence data, the difference of DEMs will be influenced by interpolation in this areas. In case of comparable areas, the differences of DEMs can be calculated with no additional consideration. The proposed method is based on the creation of a voxel structure in the two data sets. A first occupancy analysis is used to mark areas which are outside of the water areas of the reference and will mark uncaptured data in this "dry" areas. Inside the water areas of the reference, a histogram of heights of all points inside a single voxel is used to distinguish between ground points and water points. Points corresponding to the lowest height values are marked as ground and points corresponding to the next confidence interval above the ground are marked as water. If there is no water found, the points inside the voxel are marked as "dry" points inside reference water area. If there are water points found, the refraction correction is done and a second occupancy analysis of the new ground points and the ground points of the reference used. If the new ground points are in unoccupied voxels of the reference, the new points are marked as points with no reference data otherwise the new points are marked as comparable points. Results are evaluated on a proposed benchmark data set of the Mangfall area in Bavaria, Germany. The multi temporal data consist of an ALB data set of a flight campaign at 2012 and an ALB data set at a flight campaign at 2017. Furthermore, there was a change in the scanning system between this two data sets. The flight campaign at 2012 was done using a Riegl VQ 820G system and the flight campaign at 2017 using a Riegl VQ 880G system. The testing area maps a region of the Mangfall in Gmund at the Tegernsee. This area is labelled manually with the classes water, ground, vegetation, building and the proposed labels for changes in water areas. Using this ground truth, the accuracy of the proposed approach is evaluated.

\footnotetext{
${ }^{*}$ Corresponding author
} 


\section{STATE OF THE ART}

Sensors for airborne LiDAR bathymetry (ALB) are evolved in the last decades. Today, they are widely used in the measurement of large scale shallow water areas, e.g. rivers (???).

One important attribute of monitored river areas is the change of river ground points. This is used to get information about habitats or sediment transportation (??). To create change maps of these ground point changes the common method is to calculate differences of DEMs and specify significant differences with a statistical test (??). This method is able to distinguish between geometric changes and height differences caused by measurement errors. This DEMs are generated based on the river ground points which must be corrected due to light refraction effects on the water surface. Therefore, the extraction of water models plays an important role in the analysis of ALB data as well. The separation between water and ground can be performed either semi-automatic (?) or by analysing full waveform data (?).

In practical application it can either happen that there is no water return or the laser signal cannot penetrate the water body until the ground. By considering mutli-temporal data the turbidity and absorption grade of the water body can also change. Therefore, it can either happen that there is no ground in the reference or there is no water return in actually wet areas. This two cases should be considered in the change detection to determine different variances of significant changes. Furthermore, marking areas where are no water echoes in the new data can be used to try to get more echoes of the waveform analysis in post processing. Therefore, the semantic change labels of the proposed method could be used to determine areas where waveform data should be analysed with a different model or to determine different thresholds for significant changes.

\section{METHOD}

The first step of the proposed approach is an occupancy analysis. A voxel structure is defined for the reference point cloud and the new point cloud. These voxel structures are initialised with the same size and are calculated to the same level of detail. Therefore, a single voxel in the new point cloud can be matched to a single voxel in the reference point cloud. If the voxel in the new data is occupied but unoccupied in the reference data, the points inside the new voxel are marked as new points and therefore get the label "changed". Furthermore, the occupancy analysis is used to transfer the semantic information from the reference to the new data. If the voxel in the reference and in the new data are occupied, the median of the classes inside the reference voxel is assigned to the points inside the new voxel.

Furthermore, the semantic reference is used to define water areas in the new data. New points inside the area of the reference water model are considered as points inside river areas. Voxels consisting of points inside river areas are used to generate a height histogram. This height histogram is used to define ground and water points in the new data. Points corresponding to the lower confidence are marked as ground points and points corresponding to the next confidence are marked as water points. This is shown in Figure 1. The confidences are searched starting at the left side of the histogram, where one confidence is defined by connected bins holding information. The first bin which holds information marks the beginning and the next bin without information marks the end of one confidence. All bins of one confidence are then
Data: semantic reference data and new data

Result: new data with semantic informations $\mathrm{V} 1$ = calculate voxel structure(reference); $\mathrm{V} 2$ = calculate voxel structure(new data);

foreach voxel $v \in V 2$ do

if $v \in$ waterModelReference then

$\mathrm{h}=$ histogramOfHeights(v.points); assignClassToPoints(v,h);

end

else

vref $=$ getCorresponding $\operatorname{Voxel}(\mathrm{v}, \mathrm{V} 1)$;

if $v$ ref $==$ occupied then

assignClassToPoints(v,medianClass(vref.points));

end

else

assignClassToPoints(v,"change"); end

end

end

Algorithm 1: Algorithm summarise assigning class labels

used to calculate the mean and variance of the confidence interval.

Data: voxel and histogram of heights

Result: assigned ground and water labels

groundFirstBin $=h$.firstWhere $(0$, number $>0)$;

groundLastBin $=$ h.firstWhere $($ groundFirstBin, number $<1)$;

waterFirstBin $=$ h.firstWhere $($ groundLastBin, number $>0)$;

waterLastBin $=$ h.firstWhere $($ waterFirstBin, number $<1)$;

averageG,sdtG =

averageAndVariance(groundFirstBin,groundLastBin);

averageW,sdtW =

averageAndVariance(waterFirstBin,waterLastBin);

foreach point $p \in$ voxel.points do

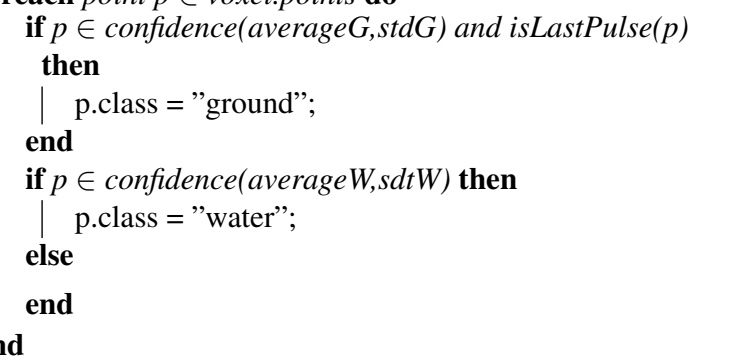

Algorithm 2: Algorithm summarise divide ground and water

The water points of the new data are used to calculate the water model of the new data and to correct the ground points in river areas. If no correction is possible due to missing water points, the points are marked as not corrected. After successful correction the river ground points are used for a second occupancy analysis. If the new point is inside an occupied voxel in the reference, the point is marked as comparable. If the river ground point is in an area which is not occupied by reference data, the label for "no reference" is assigned. Therefore, the label for not corrected points shows points inside areas where in the reference data was a recognition of water areas but in the new data was not. This assign of change labels based on a decision tree is shown in figure 2. This decision tree only looks at ground or new points in river areas. Since the histogram based voting for ground and water have a higher focus to ground points, the labels ground and change are the main labels in river areas. 


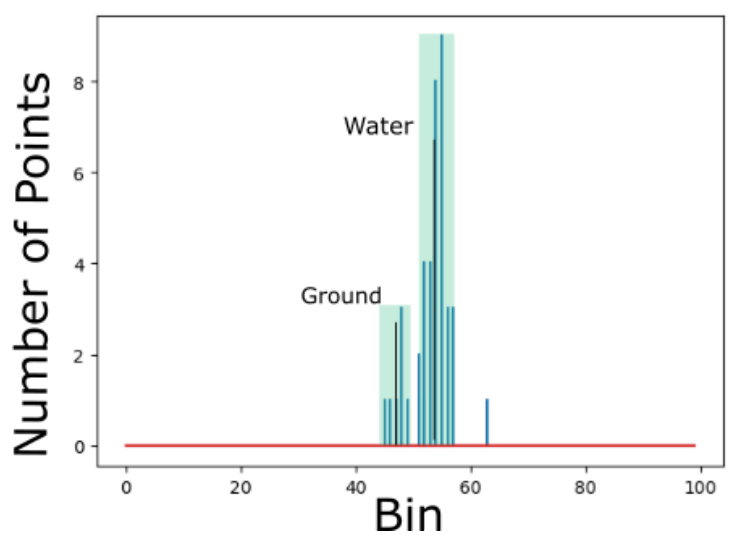

Figure 1. Schematic histogram based classification into ground and water points

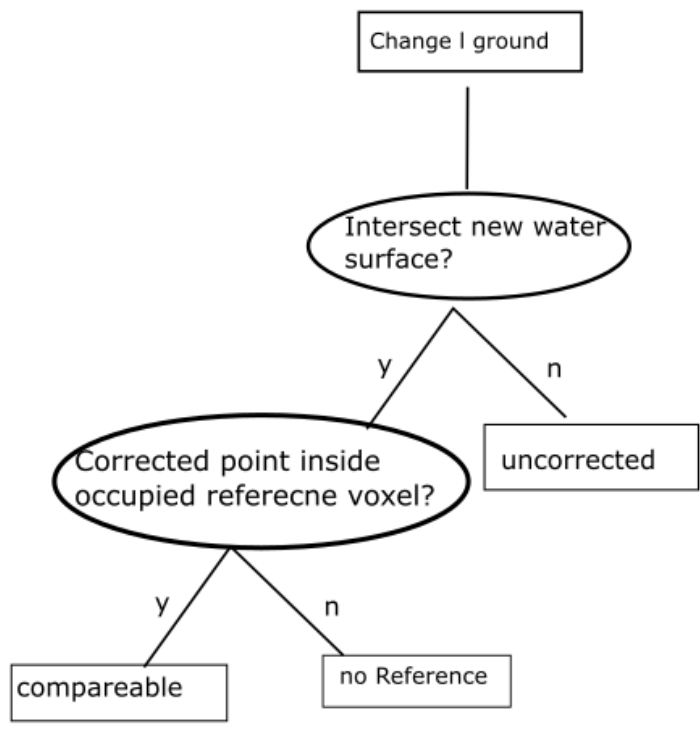

Figure 2. Scheme of the decision tree for assigning change labels. Input are the ground points and new points in the river area.

\section{DATA}

The proposed approach is evaluated using a manually labelled ground truth of the Mangfall area in Bavaria, Germany. The area is captured with airborne LiDAR during two flight campaigns, one at 2012 and another at 2017. Furthermore, there was a change in the scanning system between this campaigns which result in different resolutions of the point clouds. The dataset from 2012 was captured using a Riegl VQ $820 \mathrm{G}$ which creates a regular scan pattern with $0.5 \mathrm{~m}$ ground sampling distance. The dataset from 2017 was captured using a Riegl VQ 880G which creates a ground sampling distance of $0.1 \mathrm{~m}$ along the scanline and 0.5 $\mathrm{m}$ between two scanlines. Furthermore, the scan systems differ in the number of maximal recognisable return pulses in the laser signal. The VQ820G recognise a maximum of about 4 pulses and the VQ880G a maximum of about 8 pulses.

The chosen test area is on top of the village Gmund and shows an area of a paper industry. This area shows structural changes in the buildings of the industry as well as structural changes inside the river channel. Furthermore, there are differences in water depths between the river channels. Another difference is the amount of recognised pulses from the river ground at the two capturing times and the amount of recognised water pulses. Therefore, all change classes are present in the chosen testing side.

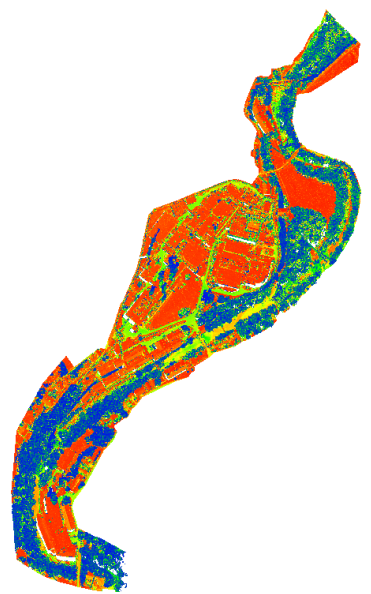

(a)

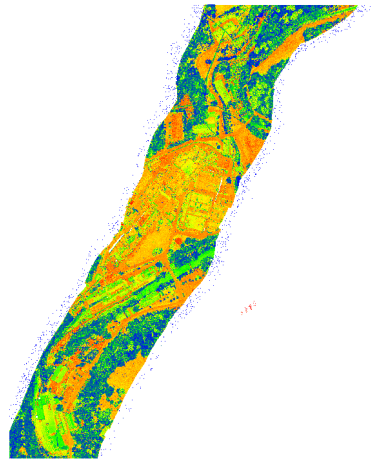

(b)
Min

\section{Intensity}

Figure 3. Overview of the LiDAR data of the test side. (a) data from 2012, captured with a Riegl VQ 820G, (b) data from 2017, captured with a Riegl VQ 880G.

Figure 3 shows an overview of the chosen area as intensity data of the scan systems.

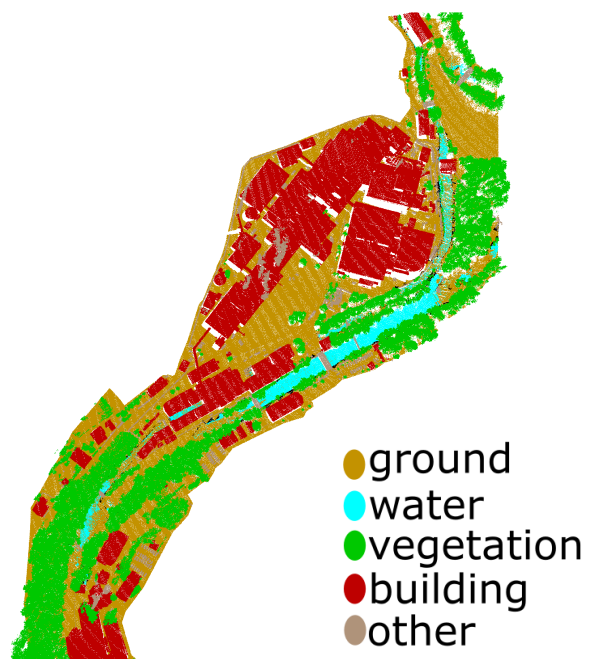

Figure 4. Overview of the semantic labels from the data of 2012, manually labeld.

The semantic information of the data from 2012 are shown in figure 4 , these semantics are manually labelled. The change labels of the dataset of 2017 are shown in figure ??. These labels are also manually created. It structural changes of buildings can be seen at the new building at the bottom of the figure and some smaller changes at the industry buildings. Especially facades and tree stems shows the areas of newly measured points. The area on the right of the figure shows an area where no water pulses in the new dataset are recognised. 


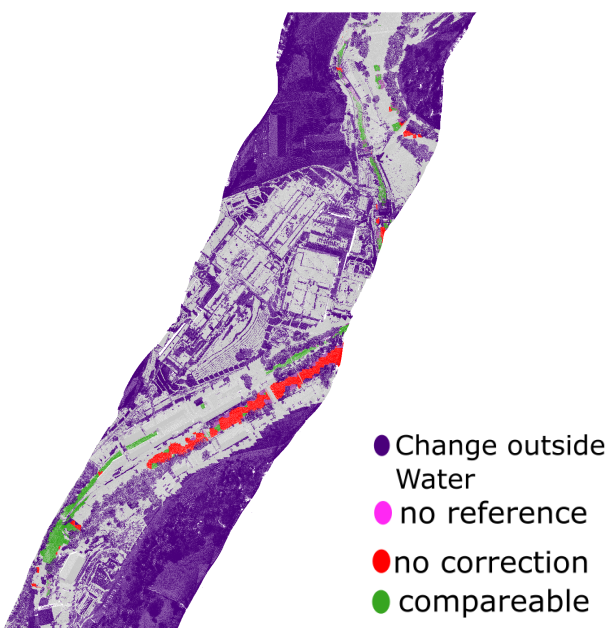

Figure 5. Overview of the semantic changes from the data of 2017, manually labeld.

\section{RESULTS}

Since the proposed approach is based on a voxel structure, the main influence to the accuracy is seen by the chosen voxel size. The voxel size should be chosen dependent on the expected ground sampling of the data. Choosing the voxel size too small has no benefit against a point wise processing and choosing the voxel size too big will create visible artefacts for the classification. Furthermore, there is a difference for the voxel structure in case of the first step of class assigning and in case of the search for water and ground points in river areas. In the latter the voxels are chosen to be not squared to hold more points along the height direction. Therefore, this paper evaluates the influence of different voxel sizes to the final accuracy of change detection.

The evaluation is separated into two parts, the first, which focus on the detection of ground and water points and the second which focus on the assign of final change labels. The first detection is more critical because it directly influences the final output of the proposed method. Furthermore, the influence of different classification accuracy in case of ground and water points for the change labels should be evaluated too.

The first evaluation focuses on the accuracy of the assign of reference labels to the new data set. with a used voxel size of $1 \mathrm{~m}$ and a ground resolution for the height histogram of $3 \mathrm{~m}$ the confusion matrix shown in table 1 was created. The evaluation focuses on the classes change, ground and water because of their influence to the determination of change labels.

\begin{tabular}{c|ccc} 
GT & change & ground & water \\
\hline change & 0.25 & 0.20 & $3 \mathrm{e}-03$ \\
ground & 0.19 & 0.35 & $5 \mathrm{e}-03$ \\
water & $2 \mathrm{e}-04$ & $2 \mathrm{e}-03$ & $1 \mathrm{e}-04$
\end{tabular}

Table 1. conf Matrix preproz. 1m, enh 3m

Considering this three classes there is an unequal distribution. The unequal distribution of this classes can be seen in the confusion matrix. The confusion matrix shows also the dependencies of the classes to each other. It can be seen that the separation between the ground and change class is not clear. The most false negatives of ground points are labelled as change and also the same for the points of changes. The evaluation of the confusion matrix is shown in table 2 .

\begin{tabular}{ccccc} 
Resolution & label & TPR & TNR & acc \\
\hline \multirow{3}{*}{$1 \mathrm{~m}$} & change & 0.55 & 0.66 & 0.61 \\
& ground & 0.64 & 0.56 & 0.60 \\
& water & 0.04 & 0.99 & 0.99 \\
& OA: 0.60 & & \\
\hline \multirow{3}{*}{$2 \mathrm{~m}$} & change & 0.43 & 0.81 & 0.64 \\
& ground & 0.80 & 0.43 & 0.63 \\
& water & 0.04 & 0.99 & 0.99 \\
& OA: 0.63 & & \\
\hline \multirow{3}{*}{$4 \mathrm{~m}$} & change & 0.25 & 0.92 & 0.65 \\
& ground & 0.61 & 0.60 & 0.60 \\
& water & 0.02 & 0.99 & 0.99
\end{tabular}

Table 2. Table of assigned classes

The table shows that all classes have a high sensitivity (TNR) and accuracy (acc). Especially the class for water points reaches a very high sensitivity which indicates that the approach is able to distinguish between water and the other classes. However, the true positive for the water class is very low, which indicates the wrong assignment of water points. Due to the unequal distribution of the classes, the recall (TPR) and the sensitivity have extremely different values.

The influence of the chosen voxel size can be seen by degreasing TP rates of the label change while the sensitivity increases. Choosing the voxel size to greater value will increase the amount of overlap and therefore marks fewer points as changed areas. This is the reason why the TP rate falls. By assigning the ground label the higher overlap results in more assigned ground points which results in a higher TP rate. However, there are also more false assigned ground points due to the higher sampling of the voxel structure. Therefore, the size of the voxels affects the sensitivity in a negative way. The assign of water labels is in comparison independent on the voxel size. This can be explained by the use of the histogram of assignment of final water labels. The horizontal resolution of the voxels used for histogram calculation is chosen to be fix at $3 \mathrm{~m}$. Choosing smaller voxels will miss some water points and choosing bigger voxels will return into histograms where no separation between the significance areas is possible.

The second evaluation goes for the assignment of change labels. This is also evaluated for different voxel sizes on base of the assigned classes. The use of the assigned classes from $1 \mathrm{~m}$ voxel resolution or from $2 \mathrm{~m}$ voxel resolutions show vanishing influences. Therefore, the evaluation of the change labels is further discussed in this paper on the example of assigned labels of $1 \mathrm{~m}$ voxels. an example confusion matrix with a voxel size of $1 \mathrm{~m}$ for assigning the change labels is shown in table 3.

\begin{tabular}{c|ccc} 
GT & no water & no ref & compareable \\
\hline no water & 0.01 & $6 \mathrm{e}-03$ & 0.01 \\
no ref & $3 \mathrm{e}-03$ & $2 \mathrm{e}-03$ & $4 \mathrm{e}-03$ \\
compareable & $5 \mathrm{e}-03$ & $4 \mathrm{e}-03$ & 0.02
\end{tabular}

Table 3. conf Matrix preproz. 1m, enh 3m, change $1 \mathrm{~m}$

compared to the classes, the change classes are more equally distributed. Furthermore, the candidate for false assignment are the 
class "comparable", which is assigned to "no water". This false assignment is also dependent on the chosen voxel size which is used for water model interpolation. The false assignment of other change classes is vanishing compared to the total amount of points. The evaluation of the confusion matrix is shown in table 4.

\begin{tabular}{|c|c|c|c|c|}
\hline Resolution & label & TPR & TNR & acc \\
\hline \multirow[t]{4}{*}{$0.5 \mathrm{~m}$} & no water & 0.56 & 0.96 & 0.94 \\
\hline & no ref & 0.08 & 0.98 & 0.97 \\
\hline & compareable & 0.41 & 0.95 & 0.93 \\
\hline & \multicolumn{2}{|c|}{ OA: 0.88} & & \\
\hline \multirow[t]{4}{*}{$1 \mathrm{~m}$} & no water & 0.37 & 0.98 & 0.96 \\
\hline & no ref & 0.13 & 0.98 & 0.97 \\
\hline & compareable & 0.54 & 0.92 & 0.91 \\
\hline & \multicolumn{2}{|c|}{ OA: 0.87} & & \\
\hline \multirow[t]{4}{*}{$2 \mathrm{~m}$} & no water & 0.20 & 0.99 & 0.96 \\
\hline & no ref & 0.18 & 0.97 & 0.96 \\
\hline & compareable & 0.61 & 0.89 & 0.88 \\
\hline & \multicolumn{2}{|c|}{ OA: 0.84} & & \\
\hline \multirow[t]{4}{*}{$4 \mathrm{~m}$} & \multirow{4}{*}{$\begin{array}{c}\text { no water } \\
\text { no ref } \\
\text { compareable } \\
\text { OA: } 0.8\end{array}$} & 0.63 & 0.93 & 0.92 \\
\hline & & 0.08 & 0.98 & 0.97 \\
\hline & & 0.34 & 0.92 & 0.89 \\
\hline & & & & \\
\hline
\end{tabular}

Table 4. Table of change classes, preproz $1 \mathrm{~m}$

The evaluation shows a very high selectivity and accuracy for all change classes. Therefore, the selection of the river areas out of the total amount of points is accurate with the use of the reference water model. However, the TP rate shows some differences between the change classes or between different used voxel sizes. Furthermore, the TP rate as well as the overall accuracy show that the classification works well for selecting seed points. The classified water points are used for interpolating a water model. Therefore, the weak classification of the water points still works good enough to distinguish between the change classes.

Choosing higher voxel sizes will result in a decreasing TP rate of the "no water" class but an increasing TP rate of the class "comparable". The constant selectivity value at different sizes show that the TP rates results from a lower false assignment of the class "comparable". Considering the calculation of the new water model, the higher voxel size will result in a greater neighbourhood for interpolation. Therefore, more gaps are filled and more intersection points are calculated which leads to smaller areas of "no water" points. Table 4 shows also the effect of big interpolation neighbourhoods by the voxels size of $4 \mathrm{~m}$. If the interpolation neighbourhood is too big, the resulting resolution of the voxel structure will lead to more false assignments as in the case of the class assignment.

The ground truth for the semantic labels in the new data is shown in figure 6. Figure 7 shows the results of assigning the reference labels. Furthermore, figure 7 shows the influence of different voxel sizes. It can be seen that bigger voxel resolutions result into more homogeneity inside the class but also to expanding class borders. The main classes to focus on for the change detection are the classes ground and water which can be successful detected. There are very few water echoes in the new data set but the proposed histogram based method is able to find good seeds even at a sparse distribution of water echoes.

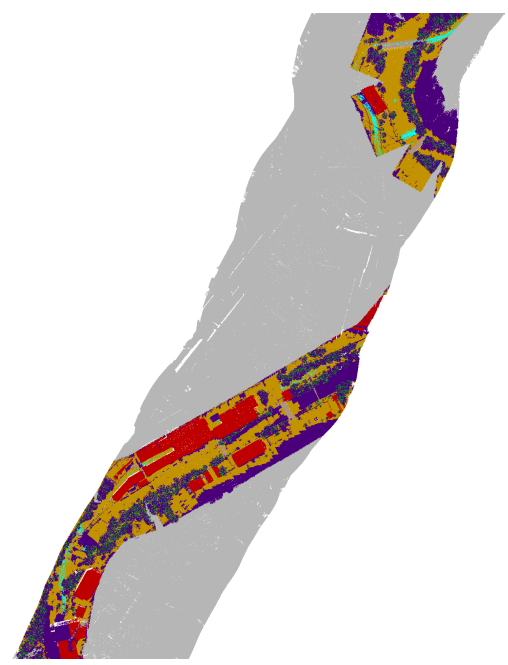

Figure 6. GT for assigned labels.

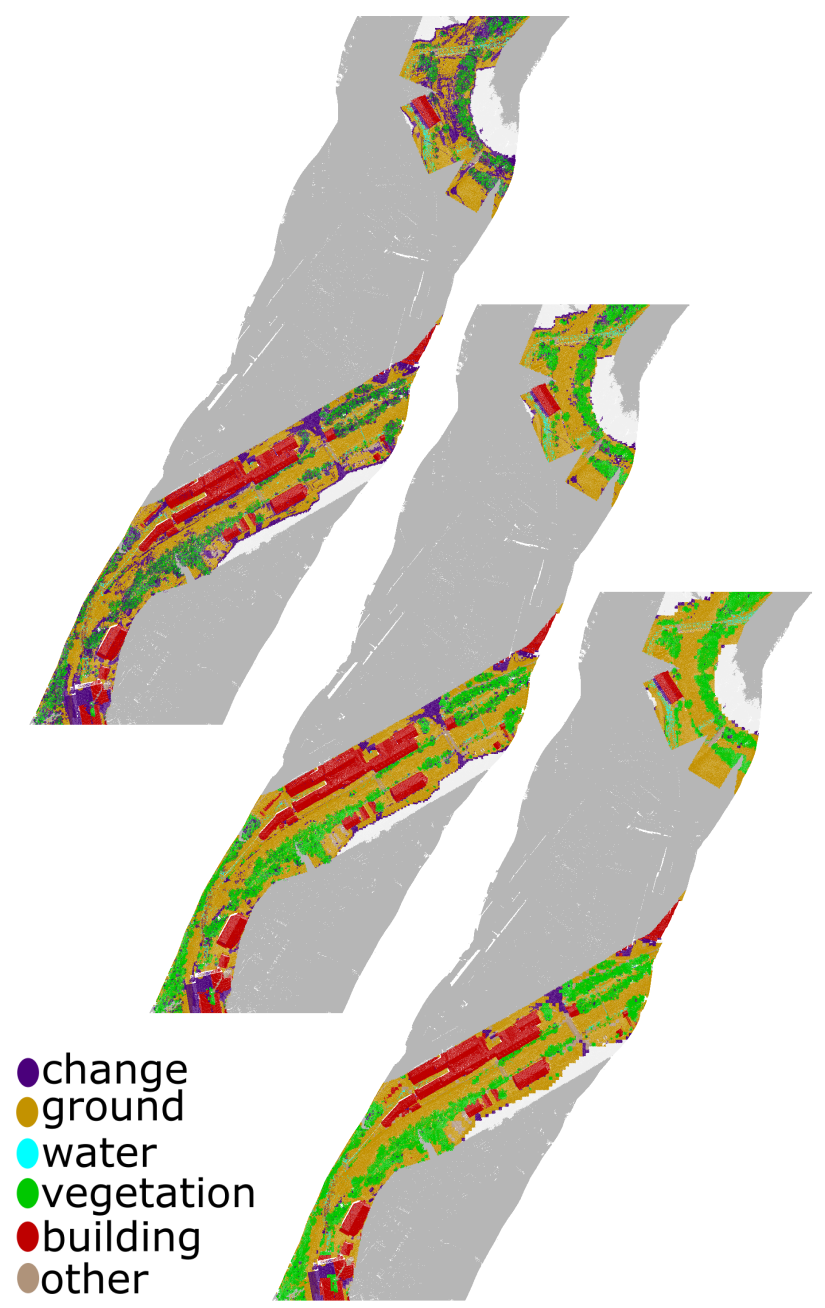

Figure 7. Result for assigning reference labels. Shown are the results of different voxel sizes, from top to down: $1 \mathrm{~m}, 2 \mathrm{~m}, 4 \mathrm{~m}$.

The Ground truth of the change labels can be seen in figure 8 . The most appearing labels are "comparable" and "no water".

Figure 9 shows the influence of different voxel sizes for the assignment of these change labels. The voxel resolution influences the neighbourhood of points to which are the labels assigned as well as the interpolation neighbourhood for the water model. The 


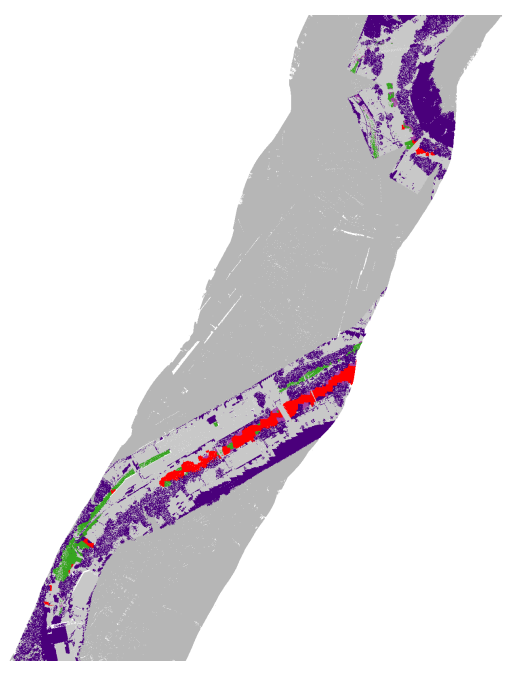

Figure 8. GT for assigned labels.

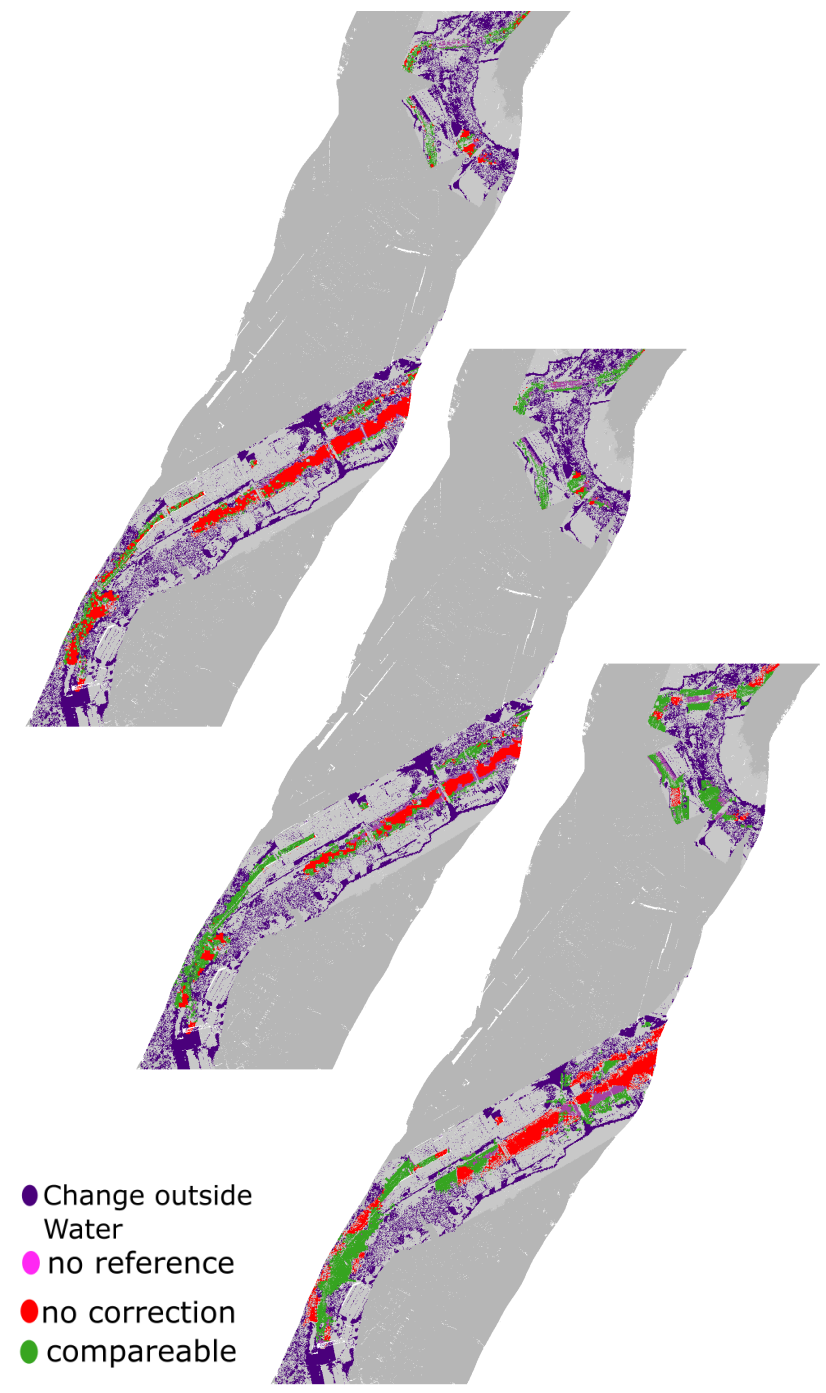

Figure 9. Result for assigning change labels. Shown are the results of different voxel sizes, from top to down: $0.5 \mathrm{~m}, 1 \mathrm{~m}, 4$ $\mathrm{m}$.

influence of the water model can be seen by a higher amount of no water points. Due to the sparse distribution of water points the water model gets sparse, too. The sparse model leads to a lower amount of intersections, which can be seen by the count of "no water" labels in the case of small voxels. The case of a voxel size of $1 \mathrm{~m}$ shows the influence of interpolation for the water model. The sparse distributed seeds of water points are used to calculate an interpolated model which leads to shrinking amount of no water points. Only the areas which have no seeds are still marked as no water. Considering the larger voxel size, the influence of the neighbourhood for assignment is seen by the footprints of the voxels at the class borders.

\section{CONCLUSION}

This paper proposes a method to create semantic information about change areas in river beds. These semantics include comparable regions, regions without reference data and regions without water echoes in reference water areas. The proposed method uses semantic information of reference data to distinguish between areas outside of water areas and the water areas. A voxel structure is used to check for occupied voxels inside the reference and the new data. Occupied voxels in both data are used to transfer the semantic information of the reference to the new data. At the same step, new points in the new data set can be labelled as change if they correspondent to unoccupied voxels in the reference. The water points of the reference are used to determine water areas in the new data where a histogram based approach is used to distinguish between water and ground points. Choosing bigger voxels for the transfer of semantic information will lead to expanding class borders. In contrast, choosing smaller voxels will lead to missing class assigns and therefore more false positives for changes.

For the second step, the new assigned water points are used to generate the new water model and to check for intersection of the corresponding laser beams of river ground points to the new water model. If no intersection is found, the river ground point below the old water model is marked as "no water". If reference ground points are found near to the new ground point, the point is marked as "comparable" else as "no reference". Evaluations show that even with a weak classification, high accuracies of the change labels are reachable if good seeds of ground and water points are found. The change labels reach an overall accuracy of more than 87 percent even with an overall of 60 percent for the classification. The voxel size should also be chosen not too small and not too big. Bigger voxels have the same effect of expanding class borders like in case of the transfer of semantic information. Smaller voxels lead to smaller interpolation neighbourhood for the water model and therefore a lower count of intersections.

The change labels could be used in the future to support a significance check for DEM differences. The label "comparable" marks areas which are safe to process with a difference of DEMs. The label "no reference" can be seen as additional information where interpolation for the DEM calculation happens and therefore the calculated geometry can be different to the true one. The most critical areas are the ones of the label "no water", for the test of significance, the variance of the point coordinates should be considered as higher, because of the light refraction. But if information about the sediment transport and the geometry are needed, these areas should be used to look for additional water points.

\section{ACKNOWLEDGEMENT}

This research is funded by Bayerische Forschungsstriftung, project "Schritthaltende 3D-Rekonstruktion und -Analyse (AZ-1184-15)", 
sub project "Änderungsdetektion in Punktwolken". The authors would like to thank our project partner SteinbacherConsult for providing the ALS dataset.

\section{REFERENCES}

Mandlburger, G., Hauer, C., Wieser, M., Pfeifer, N., 2015. Topo-bathymetric LiDAR for monitoring river morphodynamics and instream habitats - a case study at the pielach river. Remote Sensing, 7(5), 6160-6195.

Mandlburger, G., Pfennigbauer, M., Steinbacher, F., Pfeifer, N., 2011. Airborne hydrographic LiDAR mapping - potential of a new technique for capturing shallow water bodies. In: Proceedings of the 19th International Congress on Modelling and Simulation, Perth, Australia, 12-16.

Pfennigbauer, M., Steinbacher, F., Ullrich, A., Aufleger, M., 2010. A novel approach to laser-based hydrographic data acquisition. In: European LiDAR Mapping Forum, Salzburg, Austria.

Schwarz, R., Mandlburger, G., Pfennigbauer, M., Pfeifer, N., 2019. Design and evaluation of a full-wave surface and bottomdetection algorithm for lidar bathymetry of very shallow waters. ISPRS Journal of Photogrammetry and Remote Sensing, 150, $1-10$.

Steinbacher, F., Pfennigbauer, M., Aufleger, M., Ullrich, A., 2012. High resolution airborne shallow water mapping. In: International Archives of the Photogrammetry, Remote Sensing and Spatial Information Sciences, Proceedings of the XXII ISPRS Congress, 39, p. B1.

Wheaton, J.M., Brasington, J., Darby, S.E., Sear, D.A., 2010a Accounting for uncertainty in dems from repeat topographic surveys: improved sediment budgets. Earth surface processes and land forms: the journal of the British Geomorphological Research Group, 35(2), 136-156.

Wheaton, J.M., Brasington, J., Darby, S.E., Merz, J., Pasternack, G.B., Sear, D., Vericat, D., 2010b. Linking geomorphic changes to salmonid habitat at a scale relevant to fish. River research and applications, 26(4), 469-486. 